\title{
Two stages of learning by children and adults*
}

\author{
HERMAN BUSCHKE $\dagger$ \\ The Saul R. Korey Department of Neurology \\ Albert Einstein College of Medicine, Bronx, New York 10461
}

\begin{abstract}
Repeated attempts to retrieve a 20-item list, without confounding by any further presentation of items after their first recall, were used to show that verbal learning by children and adults can be analyzed in terms of two stages of (item and list) learning, amplifying previous analysis in terms of storage, retention, and retrieval from long-term storage. Two separate stages of learning are indicated by the failure of retrieval to increase until the abrupt onset of perfectly consistent spontaneous retrieval.
\end{abstract}

Repeated spontaneous recall, without any further presentation of each item after it has been recalled just once, has recently been used to show storage and retention in free recall verbal learning by subsequent spontaneous retrieval (Buschke, 1974). Such restricted presentation, which is a special case of selective reminding (Buschke, 1973), shows learning by allowing recall without further presentation to show retrieval from long-term storage (LTS). This report shows how such verbal learning can be analyzed in terms of the two separate stages of learning which are indicated, by the relatively constant (nonincremental) level of recall until the sudden onset of perfectly consistent recall.

\section{METHOD}

Restricted presentation was used to compare storage, retention, and retrieval in free recall verbal learning by 10 children (mean age $=9.4, \mathrm{SD}=1.0$ ), 10 young adults (mean age $=22.9, \mathrm{SD}=3.1$ ), and 10 older adults (mean age $=42.2, \mathrm{SD}=$ 7.7), who were paid for their participation. Each $\mathrm{S}$ was tested individually, he was read a list of 20 animals $^{1}$ at a 2 -sec rate, for free recall in any order immediately after presentation. After the initial presentation of the entire list, the $S$ was reminded only of those items which he had not yet recalled at all, before he again tried to recall all of the items in the entire list. On each of the 12 recall trials, the $S$ always tried to recall all of the items, even though items were never presented again after each was recalled just once. There were hardly any presentations after the fourth recall trial, since almost all items had been recalled at least once by then. In order to obtain the maximum spontaneous retrieval from LTS necessary for accurate evaluation of storage and retention, the Ss were encouraged to extend recall even after it became difficult, and were allowed as much time as they needed to achieve maximum recall on each trial. ${ }^{2}$

\section{RESULTS AND DISCUSSION}

\section{Storage, Retention, and Retrieval}

Storage is shown by the spontaneous retrieval of an item without any further presentation, indicating initial

*This work was supported by USPHS Grants MH-17733 to $\mathrm{H}$. B. From NIMH, NS-03356 from NIMS, and HT-01799 from NICHD. I thank Christine Hiney, Katie Buschke, and Tom Buschke for experimental assistance.

†Requests for reprints should be sent to: H. Buschke, M. D., Albert Einstein College of Medicine, 1300 Morris Park Avenue, Bronx, New York 10461. storage of (information about) that item on (or before) the trial of initial recall (which is the last time that item was presented). Spontaneous retrieval of an item without any further presentation after its initial recall shows retention of (information about) that item in long-term storage despite any retrieval failure after its initial recall (Buschke, 1974). Therefore, the cumulative number of items stored provides an estimate of the number of items in storage on each trial during learning. Retrieval from LTS is shown directly by the number of items recalled without presentation on each trial; recall without presentation indicates retrieval from long-term storage because it shows recall despite interference due to the presentation and recall of other items (Glanzer, 1972).

Figure 1 shows storage, retrieval, and consistent retrieval (list learning ${ }^{3}$; Buschke, 1973), as well as the total number of items recalled (left) which shows slower learning by the older adults and children than by the younger adults. The lower total recall of these children appears to reflect mostly their greater difficulty in retrieval, since they stored (information about) almost all of the items. Although these children stored almost as many items as the young adults, their retrieval on each recall trial was much less than retrieval by the young adults. The older adults also retrieved less than the young adults. The still lower consistent retrieval by the children and older adults indicates their slower list learning. ${ }^{3}$

Figure 2 shows excellent retention in LTS by the children and older adults, as well as by the young adults, despite more recall failures by the children and older adults. This is shown by the small number of recall failures which were not recovered again (total lost). Although the children and older adults had more recall failures, they also recovered most of their recall failures again spontaneously.

\section{Two Stages of Learning}

Even though such verbal learning does appear to be limited more by retrieval than by storage or retention, it is possible that retrieval might just reflect the adequacy of storage. Therefore, it would be appropriate to analyze 



Fig. 1. Storage and retrieval in free recall learning by children, young adults, and older adults (shown by spontaneous' retrieval without any further presentation of items recalled once).



Fig. 2. Retention in free recall learning despite more recall failures by children and older adults than young adults, shown by the small number of items never recovered again (total lost).

such verbal learning in some other way which does not involve the distinction between storage and retrieval; this can be done by an analysis in terms of stages of learning. This paradigm for verbal learning allows us to determine that some items can be consistently retrieved from LTS on all subsequent recall attempts without any further presentation at all, separating retrieval from LTS into consistent retrieval and inconsistent or random retrieval. Figure 3 shows that the probability of such random retrieval of an item from LTS does not increase prior to the abrupt onset of consistent retrieval of that item from LTS. The finding that the probability of (random) retrieval from LTS remains relatively constant until the abrupt onset of consistent retrieval indicates that random retrieval and consistent retrieval from LTS probably represent two different stages of learning (Kintsch \& Morris, 1965; Restle, 1965), and supports the contention that consistent retrieval from LTS indicates that an item has been learned as part of a list (so that it can be consistently retrieved because its retrieval has been integrated with the retrieval of other consistently retrieved items). The number of items recalled on successive recall attempts during such verbal learning seems to increase because (information about) more and more items are encoded in the second stage of learning for consistent retrieval from LTS, ${ }^{4}$ not because the probability of recalling each item increases.

A more explicit account of how recall increases during verbal learning, in terms of these stages of random retrieval and consistent retrieval from LTS would include the following components: the number of items initially encoded and retained in the first stage of (item) learning (for randorn retrieval from LTS), the number of items initially encoded and retained in the second stage of (list) learning (for consistent retrieval right from their initial recall), the relative probability of random retrieval from the first stage of (item) learning, and the rate at which items are transferred from the first stage of (item) learning to the second stage of (list) learning (additional list learning). ${ }^{4}$

Figure 4 shows such an analysis of free recall verbal learning by these children, young adults, and older adults in terms of the two stages of (item and list) learning. The young adults initially encoded 13.6 of the 20 items for consistent retrieval from LTS (initial list learning) and relatively few in the first stage of random

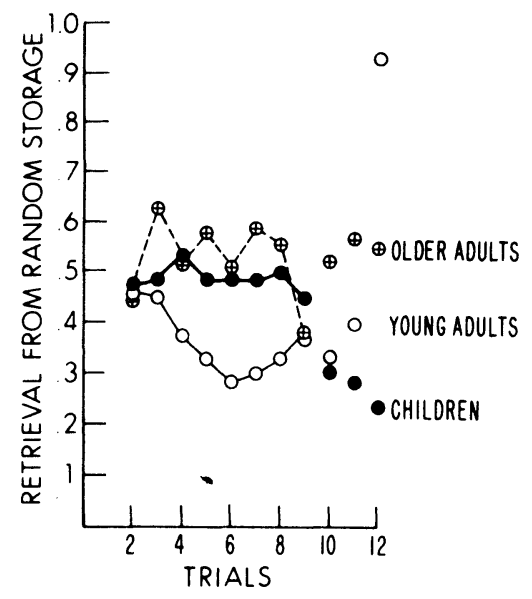

Fig. 3. Nonincremental probability of random retrieval from long-term storage (prior to onset of consistent retrieval). 
Fig. 4. Analysis of verbal learning in terms of stages of random retrieval (item learning) and consistent retrieval (list learning) from long-term storage, to show random (item) storage, nonincremental retrieval from random storage, initial consistent storage and retrieval (list learning), and transition from random to consistent retrieval (additional list learning.
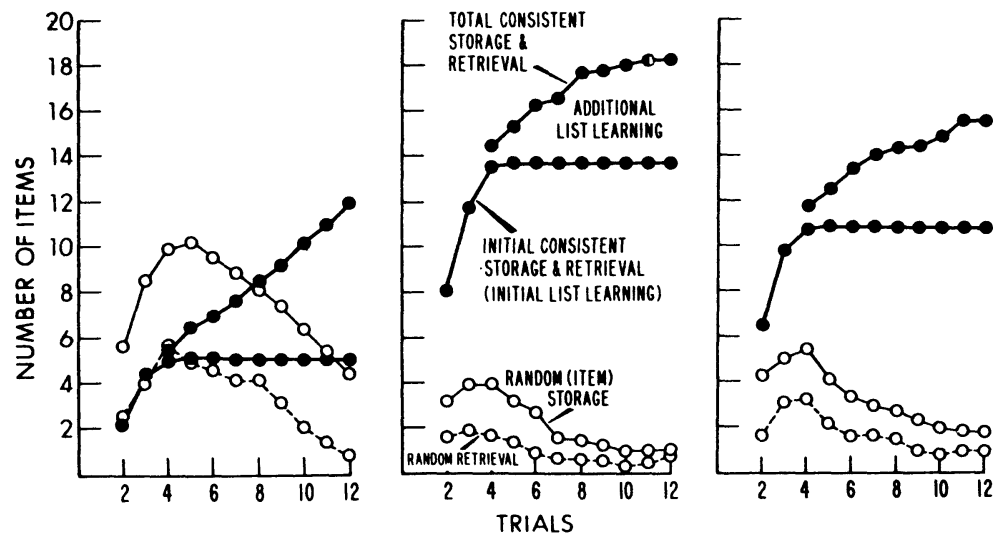

Kintsch, W. \& Morris, C. J. Application of a Markov model to free recall and recognition. Journal of Experimental Psychology, 1965, 69, 200-206.

(item) storage for random retrieval from LTS. The number of items in the first stage of item learning decreased steadily as (information about) more and more items was transferred to the second stage of consistent retrieval (list learning).

The children initially encoded only 5.2 items in the second stage for consistent retrieval from LTS (initial list learning), but initially encoded many more items in the first stage of item learning for random retrieval. However, as learning progressed these children increased their list learning by effective transfer of items to the second stage of list learning for consistent retrieval. The older adults initially learned 10.9 of the 20 items as part of the list, and initially encoded more items in random (item) storage than young adults but fewer than the initial random storage by children. The older adults also appear to have transferred items from random to consistent retrieval effectively.

Although these analyses seem to show some interesting differences in verbal learning by children and adults, the present data are not adequate for definitive evaluation of any changes in learning which might be related to development and aging (Buschke, in press). However, these findings do show two distinct stages in learning by children as well as adults (under conditions which permit the $S$ to show learning by recall without presentation), and demonstrate how analysis in terms of these stages of learnin g can provide a more explicit and illuminating account of what happens during verbal learning.

\section{REFERENCES}

Buschke, H. Selective reminding for analysis of memory and learning. Journal of Verbal Learning \& Verbal Behavior, 1973 12, 543-550.

Buschke, H. Spontaneous remembering after recall failure. Science, $1974,184,579-581$

Buschke, $\mathbf{H}$. Components of verbal learning in children: analysis of selective reminding. Journal of Experimental Child Psychology, in press.

Glanzer, M. in G. H. Bower (Ed.), Psychology of learning and motivation. Vol. 5, New York: Academic Press, 1972 Pp. 129-193.
Psychology, 1965, 69, 200-206
Restle, F. Significance of all-or Bulletin, 1965, 64, 313-315.

\section{NOTES}

1. The items in this list of animals were: dog, fox, horse buffalo, lion, rhinoceros, elephant, antelope, bear, lamb, rat, raccoon, sheep, llama, goat, cheetah, squirrel, beaver, donkey, turtle.

2. To justify the extra effort of extended recall and of repeated recall attempts without further presentation of items after they have been recalled once, the Ss must learn as soon as possible that they really can increase their retrieval in this way. This paradigm encourages the $S$ to try to recall all items in the entire list from the onset, rather than just some self-selected part of the list. Since children appear to have difficulty in remembering what they have already recalled verbally on a given recall attempt, they were allowed to repeat themselves, in order to avoid suppressing responses because they were not sure if they had already recalled them. This seems appropriate, since the object is to maximize the retrieval of as many different items as possible.

3 . When a $S$ can recall all of the items in a list on every recall attempt, without any further presentations at all, we say that the $S$ has learned the (entire) list. Therefore it seems reasonable to say that when a $S$ has learned to recall $50 \%$ of the items consistently on all recall attempts, without any further consistently on all recall attempts, without any further
presentation of those items, then $50 \%$ of the list has been learned. It appears that consistent retrieval of an item on all subsequent recall attempts, without further presentation, indicates that item has been learned as part of the list; i.e., its retrieval has been integrated with the retrieval of those other items that also are consistently retrieved, to that its retrieval is no longer affected by output interference. Therefore the cumulative number of those items which are consistently retrieved on all subsequent recall attempts without any further presentation should provide a reasonable estimate of list learning. A good feature of this measure, at least for learning by the unnecessary assumption that list learning must be reflected by an increasingly consistent order of recall.

4. A reasonable interpretation of these two stages of random and consistent retrieval from LTS is that they indicate item available in the S's permanent memory. The $S$ learns to selectively retrieve from his permanent memory just the items in the list (without omitting any and without including any other items which are not part of the list to be learned). Even though items are retrieved only inconsistently before they have been learned as part of the list, when their retrieval seems to be integrated with the retrieval of other items for consistent spontaneous retrieval, some kind of information which identifies them as correct target items is retained. It is as though items in permanent memory were first "tagged" for retrieval by random search and subsequently included in a more organized retrieval scheme.

(R eceived for publication July 25, 1974.) free recall of the items in any order, is that it does not require learning and list learning respectively. These items are already 